\title{
Influence of hydrogen on the properties of nanostructured C-Pd films for sensing applications
}

\author{
Anna Kamińska ${ }^{1 *}$, Ryszard Diduszko ${ }^{1}$, Sławomir Krawczyk ${ }^{1}$, Elżbieta Czerwosz $^{1}$, Kamil Sobczak ${ }^{2}$ \\ ${ }^{1}$ Tele and Radio Research Institute, Ratuszowa 11, 03-450 Warsaw, Poland \\ ${ }^{2}$ Institute of Physics, PAS, al. Lotnikow 32/46, 02-668 Warsaw, Poland \\ "Coresponding author: e-mail: anna.kaminska@itrorg.pl
}

\begin{abstract}
In this paper we present the results of the investigations of nanostructured C-Pd films for hydrogen sensing applications. These C-Pd films were prepared by physical vapor deposition and then annealed in an argon flow at the temperature of $500^{\circ} \mathrm{C}$. The structure and morphology of the prepared C-Pd films were investigated using transmission electron microscopy and energy dispersive X-ray spectroscopy. We studied the influence of hydrogen on the electrical properties and crystal structure of C-Pd films. It was shown that film resistance changes depended on hydrogen concentration. At lower hydrogen concentration (up to 2 vol.\%), the films response increased proportionally to $\left[\mathrm{H}_{2}\right]$, while above $2 \mathrm{vol} . \% \mathrm{H}_{2}$, it was almost constant. This is connected with the formation of a solid solution of hydrogen in palladium at lower $\mathrm{H}_{2}$ concentration and the creation of palladium hydride at higher $\mathrm{H}_{2}$ concentration. X-ray diffraction was used to confirm the formation of $\mathrm{Pd}-\mathrm{H}$ solid solution and palladium hydride.
\end{abstract}

Keywords: C-Pd films, hydrogen sensor, GIXD measurements, resistance changes.

\section{INTRODUCTION}

Hydrogen gas is considered to be one of the most promising clean energy carriers for use in fuel cells due to its efficiency and environmental friendliness. ${ }^{1}$ Hydrogen has also been widely used for conventional technology such as hydrocracking of paraffin hydrocarbons in petroleum refineries and many hydrogenation/dehydrogenation industrial processes ${ }^{2-4}$. The development and expanded use of hydrogen gas as an energy carrier and as a chemical reactant has caused an increasing demand for fast, sensitive and accurate hydrogen sensors. This is connected with the high flammability and explosiveness of hydrogen as well as a high leaking tendency because of its small molecular size ${ }^{5}$. One of the most thoroughly studied hydrogen sensors are palladium-based devices ${ }^{6-9}$. This is associated with the highly selective interaction between palladium and hydrogen, which results in changes in palladium electrical properties ${ }^{6,10}$. The interaction of hydrogen with palladium begins with the adsorption of molecular hydrogen on palladium surface ${ }^{11}$. Then the palladium atoms catalyze the homolytic dissociation of $\mathrm{H}_{2}$ to hydrogen atoms. These hydrogen atoms diffuse into Pd lattice and occupy its interstitial sites, forming a solid solution ${ }^{1,6}$. The resulting solid solution of hydrogen in palladium has higher resistance compared to metallic palladium ${ }^{11}$. If the hydrogen partial pressure in the gas phase exceeds $1 \mathrm{kPa}-2 \mathrm{kPa}$, the further incorporation of hydrogen atoms induces a phase transition from the $\alpha$ - to $\beta$-phase and the creation of palladium hydride $\left(\mathrm{PdH}_{\mathrm{x}}\right)^{12}{ }^{13}$. This leads to an increase of the lattice constant, and thus the diameter of the palladium nanograins also increases ${ }^{14,15}$.

In this paper, we studied changes in the electrical properties and the crystal structure of carbonaceous-palladium (C-Pd) nanocomposite films under the influence of hydrogen. These C-Pd films were prepared by physical vapor deposition (PVD) and then annealed in an inert gas flow at a temperature of $500^{\circ} \mathrm{C}$. Such films contain palladium nanograins embedded in the carbonaceous matrix. The carbonaceous matrix stabilizes palladium nanograins in C-Pd films, while Pd nanograins react with hydrogen, which leads to changes in the film electrical properties and structure. The structural changes occurring during hydrogen absorption were studied in situ using the X-ray diffraction method. Hydrogen sensing measurements in the $\mathrm{H}_{2} / \mathrm{N}_{2}$ mixture were performed to determine the films sensitivity and response time. We have correlated changes in C-Pd film sensitivity and response time toward various hydrogen concentrations with the changes in the film structure.

\section{EXPERIMENTAL DETAILS}

Nanostructured carbonaceous-palladium (C-Pd) films were prepared by physical vapor deposition (PVD) and then annealed under an argon atmosphere. In the PVD process the films were deposited on unpolished fused silica substrates, using two separate sources containing fullerene $\mathrm{C}_{60}$ (Sigma-Aldrich, 99.9\%) and palladium acetate (Sigma-Aldrich, $99.98 \%)^{16}$. The resulting films were annealed at a temperature of $500^{\circ} \mathrm{C}$ in an argon flow rate of $40 \mathrm{~L} / \mathrm{h}$. Depending on the technological parameters of the preparation, films with a different structure, palladium content and electrical properties were obtained. The structure of the prepared films, palladium content and distribution of the palladium nanograins were studied using a Titan 80-300 Cubed transmission electron microscope (TEM) operating at $300 \mathrm{keV}$ incident electron beam energy, equipped with an energy dispersive X-ray spectroscope (EDS). The specimens were prepared by the mechanical stripping of the C-Pd films on a 1.000 mesh grid.

$\mathrm{X}$-ray diffraction studies were carried out on the W1 beamline at Doris III synchrotron at Hasylab. GIXD (Grazing Incidence X-ray Diffraction) measurement geometry was applied due to the low thickness (up to $300 \mathrm{~nm}$ ) and low absorption of the C-Pd films. A double Si (111) crystal monochromator provided radiation with a wavelength of $1=0.154056 \mathrm{~nm}$, equal to that of the $\mathrm{K}_{\alpha 1} \mathrm{Cu}$ fluorescence line, was used. The effect of the hydrogen partial pressure on the crystal structure was studied in situ in GIXD measurements with the $2^{\circ}$ inci- 
dent angle, using a specially designed measuring holder cell with a controlled $\mathrm{H}_{2} / \mathrm{N}_{2}$ gas flow and composition.

Hydrogen sensing measurements were carried out in a special measurement set-up, described in details in our earlier paper. ${ }^{17}$ The electrical contacts to the C-Pd films deposited on unpolished fused silica substrates were made using silver paste (Conductive Silver Paint, SPI Supplies). The distance between the contacts was $10 \mathrm{~mm}$. The scheme of a sample prepared for sensing measurements showing configuration of the silver electrodes is presented in Figure 1. The films prepared in such a way were placed in a holder in a measurement chamber, with electrical feedthrough, and a gas flow inlet and outlet. Mass flow controllers were used to dilute hydrogen with nitrogen to the chosen concentrations, with a total gas flow rate of $1 \mathrm{~L} / \mathrm{min}$. Hydrogen concentration was varied in subsequent response-recovery cycles from $0.25 \mathrm{vol} . \%$ to $4.00 \mathrm{vol} . \%$. The recovery was carried out in synthetic air at a flow rate of $1 \mathrm{~L} / \mathrm{min}$. The measurements were performed at room temperature and under atmospheric pressure.

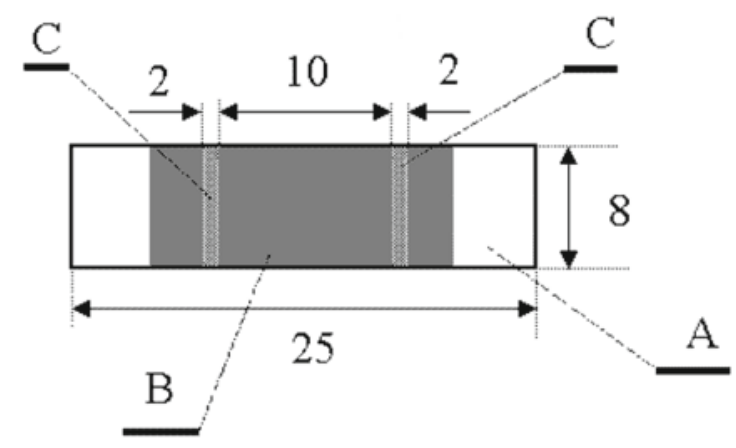

Figure 1. Scheme of C-Pd film with silver electrodes (A - fused silica substrate, B - C-Pd film, C - silver electrodes)

\section{RESULTS AND DISCUSSION}

In this paper, the results for a typical C-Pd film, obtained in a PVD process and annealed in a temperature of $500^{\circ} \mathrm{C}$, are presented. The annealing process leads to the agglomeration of palladium nanograins and the transformation of the carbonaceous matrix. The results of the TEM investigations are shown in Figure 2. The C-Pd film electronogram shown in Figure 2a confirms the presence of metallic palladium with a $f c c$ structure. The micrograph in Figure $2 b$ shows a large variation in the size and shape of the Pd nanoparticles. The

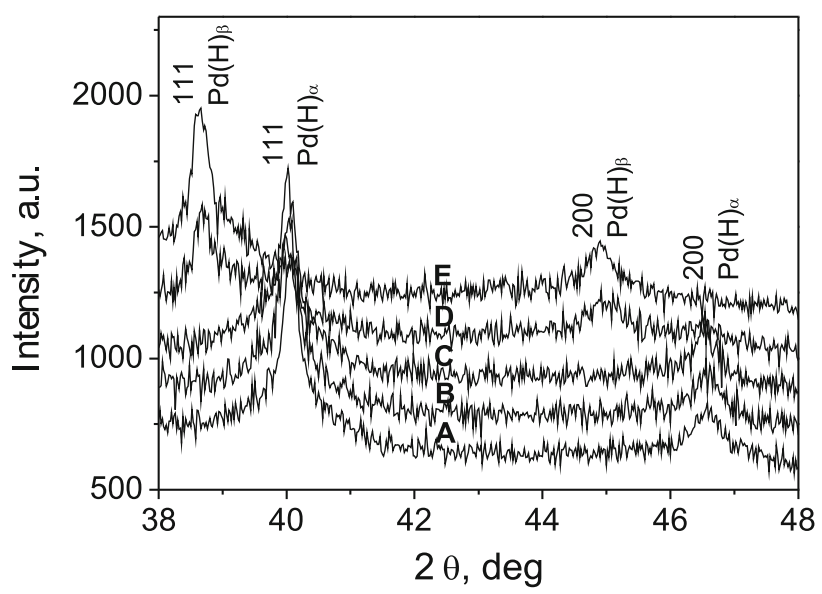

Figure 3. Diffraction patterns of the C-Pd film under the $\mathrm{H}_{2} /$ $\mathrm{N}_{2}$ atmosphere (A - 0 vol. $\% \mathrm{H}_{2}, \mathrm{~B}-0.5$ vol. $\% \mathrm{H}_{2}$, C - 1 vol. $\% \mathrm{H}_{2}, \mathrm{D}-2$ vol. $\% \mathrm{H}_{2}, \mathrm{E}-4$ vol. $\% \mathrm{H}_{2}$ )

average size of these particles is $15 \mathrm{~nm}$ and this value was obtained from fitted to lognormal distribution for 210 numbers of $\mathrm{Pd}$ nanoparticles. The histogram of the size of the palladium nanoparticles is presented in Figure 2c. The EDS spectra were collected with micron sized areas. The obtained results show that the studied C-Pd sample contains $30 \mathrm{wt} \% \pm 5 \%$ of palladium and $69 \mathrm{wt} \% \pm 5 \%$ of carbon.

Figure 3 presents the diffraction patterns of the C-Pd film, made for different concentrations of hydrogen in the $\mathrm{H}_{2} / \mathrm{N}_{2}$ gas mixture. The measuring range of diffraction angle $2 \theta$ contains two distinct diffraction peaks of the fcc palladium and palladium hydride: 111 and 200. In Figure 3, one can see the shift of the diffraction peaks to the lower diffraction angles for higher hydrogen concentration in the gas mixture. This indicates an increase of the lattice constant in the cubic cell. There are peaks of $\beta$-phase palladium hydride with the significant increased lattice constant above the 2 vol. $\% \mathrm{H}_{2}$ content in the gas mixture. The determined values of the lattice constants of the $f c c$ palladium and palladium hydride cells are summarized in Table 1.

The C-Pd film structural changes due to the hydrogen presence observed in the diffraction patterns are reflected in hydrogen sensing measurements. The sensing investigations relied on the continuous measurement of film electrical resistance while changing the gas atmosphere in the measuring chamber in subsequent response-recovery cycles. The initial resistance of the studied film in the
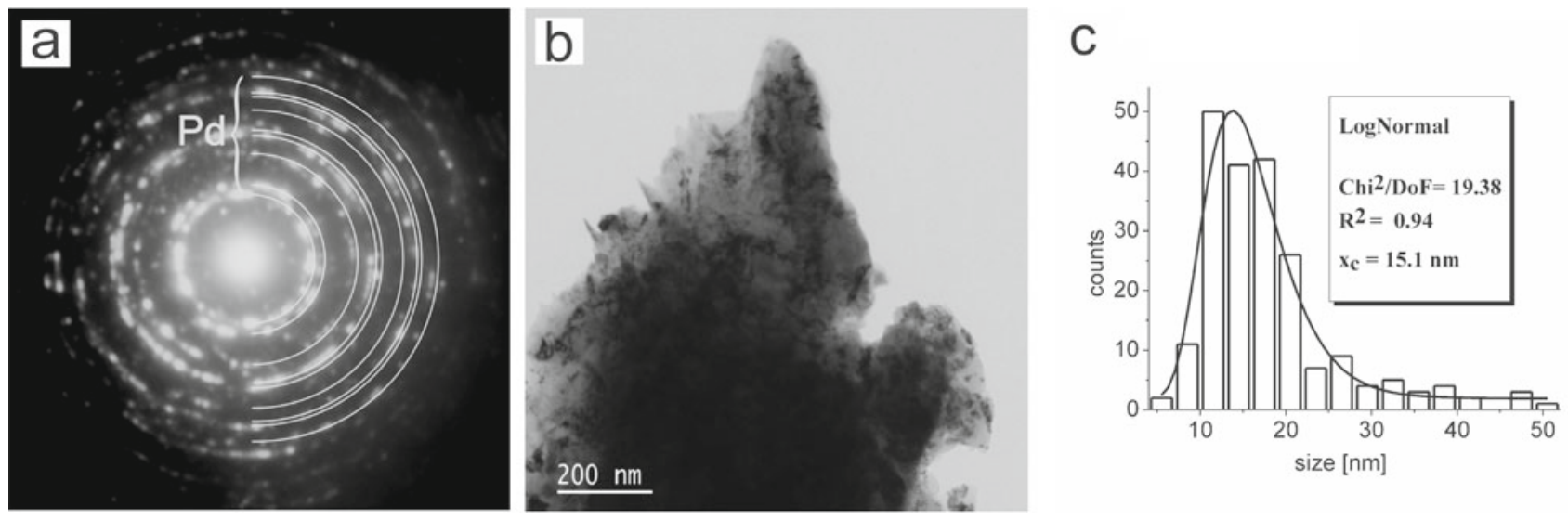

Figure 2. (a) Electronogram of selected area of the C-Pd film, (b) TEM micrograph of the same film and (c) histogram of the Pd nanoparticle size 
Table 1. Lattice constants of the Pd-H system

\begin{tabular}{|l|c|c|c|}
\hline \multirow{2}{*}{$\begin{array}{l}\mathrm{H}_{2} \text { concentration } \\
\text { [vol.\%] }\end{array}$} & \multicolumn{3}{|c|}{ Lattice constant $[\AA]$} \\
\cline { 2 - 4 } & $\mathrm{Pd}$ & $\alpha-\mathrm{PdH}_{\mathrm{x}}$ & $\beta-\mathrm{PdH}_{\mathrm{x}}$ \\
\hline 0.0 & $3.899 \pm 0.001$ & - & - \\
\hline 0.5 & - & $3.900 \pm 0.001$ & - \\
\hline 1.0 & - & $3.902 \pm 0.001$ & - \\
\hline 2.0 & - & $3.904 \pm 0.001$ & $4.030 \pm 0.002$ \\
\hline 4.0 & - & - & $4.036 \pm 0.002$ \\
\hline
\end{tabular}

air was $8.45 \mathrm{k} \Omega$. The presence of hydrogen in the gas mixture introduced into the chamber led to an increase in the film resistance. However, the introduction of the air resulted in the return of the film resistance to the initial value. The increase of the film resistance in the presence of hydrogen is connected with the formation of a solid solution of hydrogen in the palladium (at lower $\mathrm{H}_{2}$ concentrations) and the palladium hydride (at higher $\mathrm{H}_{2}$ concentrations) ${ }^{\mathbf{1 2}}$. This is consistent with our results of $\mathrm{X}$-ray diffraction measurements indicating the formation of $\mathrm{PdH}_{\mathrm{x}}$ compound. The $\mathrm{PdH}_{\mathrm{x}}$ compound has higher resistance than metallic palladium ${ }^{\mathbf{1 8}}$. The created $\mathrm{Pd}-\mathrm{H}$ system is unstable and decomposes in the air flow, causing a decrease of the film resistance. In Figure 4 the results of sensing measurements under the influence of 1 vol. $\% \mathrm{H}_{2}$ (formation of $\alpha-\mathrm{PdH}_{\mathrm{x}}$ ) and 4 vol. $\% \mathrm{H}_{2}$ (formation of $\beta-\mathrm{PdH}_{\mathrm{x}}$ ) are shown. The results are presented as a relation between relative resistance changes (percentage change in the film resistance, $\Delta R / R_{0}$ ) and time. $\Delta R / R_{0}$ was calculated from the following equation:

$\Delta R / R_{0}=\frac{R-R_{0}}{R_{0}} \cdot 100 \%$

where: $R$ - film resistance in the $\mathrm{H}_{2} / \mathrm{N}_{2}$ mixture, $R_{0}$ - the film initial resistance in the air. The magnitude of the sensor response increased with the $\mathrm{H}_{2}$ concentration in the atmosphere due to the absorption of larger amount of hydrogen. $\Delta R / R_{0}$ reached $10.4 \%$ and $19.5 \%$ after stabilization in $1 \mathrm{vol} \% \%$ and 4 vol.\% of hydrogen, respectively.

(a)
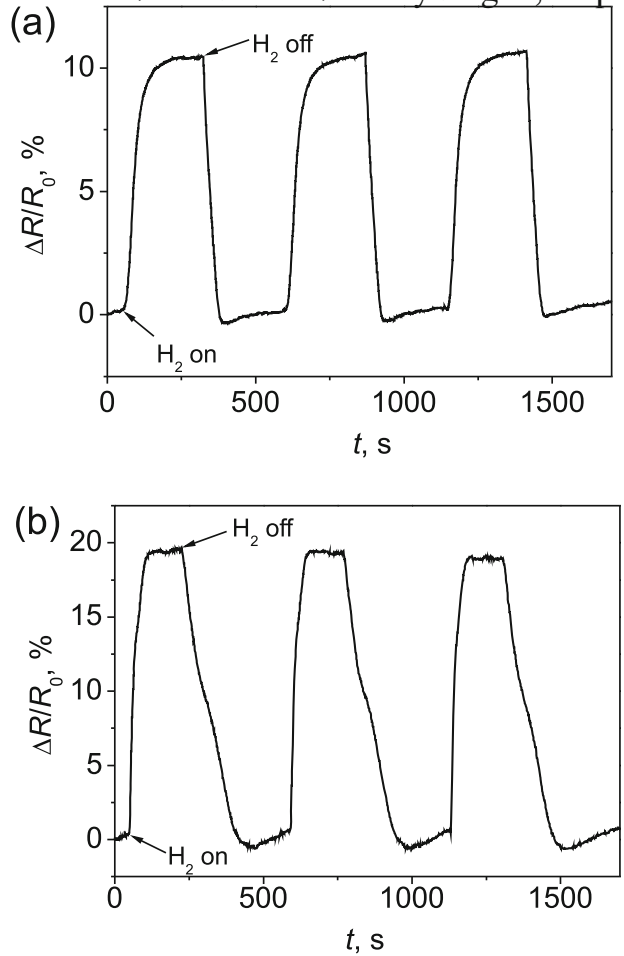

Figure 4. Changes in C-Pd film relative resistance versus time of exposure to (a) 1 vol. $\% \mathrm{H}_{2} / \mathrm{N}_{2}$ and (b) 4 vol.\% $\mathrm{H}_{2} / \mathrm{N}_{2}$
In Figure 5, the changes in the film sensitivity $\left(\Delta R_{\max }\right.$ ) $R_{0}$ ) in a function of hydrogen concentration are presented. The sensitivity is defined as a maximum relative resistance change for a given $\mathrm{H}_{2}$ concentration. It was observed that the increase in the concentration of hydrogen increased the film sensitivity. However, we can distinguish two areas on the curve of $\Delta R_{\max } / R_{0}=f\left(\left[\mathrm{H}_{2}\right]\right)$ : (1) for hydrogen concentration below 2.5 vol. $\%$, and (2) for $\mathrm{H}_{2}$ concentration range from $2.5 \mathrm{vol} . \%$ to $4.0 \mathrm{vol} . \%$. For lower concentrations of hydrogen, the sensitivity of the film varies in direct proportion to $\left[\mathrm{H}_{2}\right]$. However, after exceeding 2.5 vol.\% of hydrogen in the atmosphere, the sensitivity of the film is almost constant. This suggests that the hydrogen concentration of $2.5 \mathrm{vol} . \%$ is the limit beyond which the mechanism of interaction between hydrogen and palladium changes. For low hydrogen concentrations (below 2.5 vol.\%), the amount of absorbed hydrogen is small, so that the hydrogen forms a solid solution of $\alpha-\mathrm{PdH}_{\mathrm{x}}$ (for $\left.\mathrm{x}<0.015\right)^{\mathbf{8}}$ without causing a phase transition of palladium from the $\alpha$ - to $\beta$-phase. The formation of $\alpha-\mathrm{PdH}_{\mathrm{x}}$ causes only a slight increase in the lattice constant of palladium (Table 1). However, the absorbed hydrogen causes significant changes in the electrical properties of palladium. A linear increase in the film resistance was observed during hydrogen absorption. Similar linear relation between sample resistance and hydrogen concentration was previously observed by Lee et al. for palladium thin films ${ }^{11}$. Exceeding 2.5 vol.\% hydrogen in the atmosphere causes a palladium phase transition from the $\alpha$ - to $\beta$-phase. This results in a significant increase in the crystal lattice constants (Table 1). The increase of hydrogen concentration in the range of 2.5 vol. \%-4.0 vol.\% causes no further increase in the C-Pd film resistance. Similar results were obtained by Yang et al. in their studies of the effect of hydrogen on palladium nanowires resistance changes ${ }^{8}$. For lower hydrogen concentrations (up to 1 vol.\%), the Pd nanowires resistance increased linearly as a function of $\left[\mathrm{H}_{2}\right]$, and after exceeding 2 vol.\% of hydrogen it stabilized. Further increase in the concentration of hydrogen in the atmosphere has caused no change in the resistance of Pd nanowires ${ }^{8}$.

The dependence of the C-Pd film response time on the concentration of hydrogen is more complicated. The results of the investigations of this relationship are shown

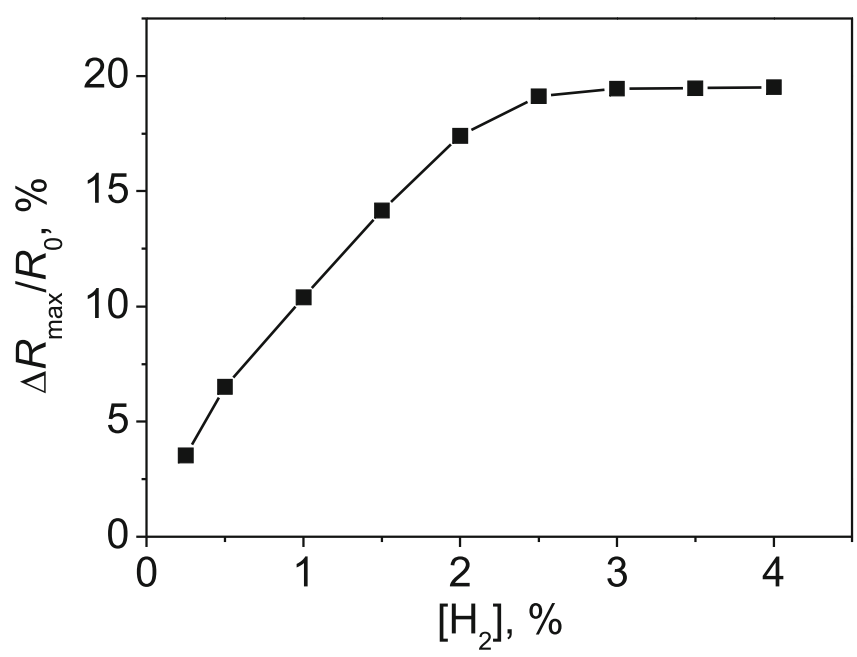

Figure 5. Sensitivity of the C-Pd film in the function of hydrogen concentration 
in Figure 6. The response time $\left(t_{90 \%}\right)$ is the time required to reach $90 \%$ of the maximum resistance change at a given hydrogen concentration. We can initially see that the response time decreased with the increasing hydrogen concentration (up to 1 vol. $\% \mathrm{H}_{2}$ ). The increase of the $\mathrm{H}_{2}$ concentration from 1 vol. $\%$ to 2 vol. $\%$ resulted in the lengthening of $t_{90 \%}$. However, a further increase of the concentration of hydrogen ( 2 vol.\%-4 vol.\%) again led to a reduction of response time. The prolonged response with an increasing hydrogen concentration from 1 vol. $\%$ to 2 vol. $\%$ is probably associated with the change of the crystalline phase. A phase transition from the $\alpha$ - to $\beta$-phase can reduce the rate of the entire hydrogen absorption process. This is consistent with the literature data of activation energy and enthalpy for processes associated with absorption of hydrogen by palladium $\left(\mathrm{H}_{2}\right.$ adsorption, $\mathrm{H}$ diffusion and the $\alpha \rightarrow \beta$ phase transition $)^{\mathbf{1 9}}$. The determined values of activation energy for hydrogen adsorption on palladium and the diffusion of hydrogen atoms are similar and they are about $5 \mathrm{kcal} / \mathrm{mol}$, while the enthalpy of phase transition from the $\alpha$ - to $\beta$-phase is about two times higher ${ }^{19}$. This means that the $\alpha \rightarrow$ $\beta$ phase transition requires higher energy than the previous steps of $\mathrm{H}_{2}$ absorption (hydrogen adsorption and diffusion). Therefore, the phase transition can slow the hydrogen absorption process at higher $\mathrm{H}_{2}$ concentrations and thus extend the film response time. As a result, there is a maximum on the curve $t_{90 \%}=f\left(\left[\mathrm{H}_{2}\right]\right)$. In our previous investigations of the kinetics of interaction of hydrogen with the C-Pd films for lower $\left[\mathrm{H}_{2}\right]$, we showed that $\mathrm{H}_{2}$ adsorption is a rate-limiting step of hydrogen absorption $^{\mathbf{1 7}}$. Our current studies indicate that for higher hydrogen concentrations, phase transition from the $\alpha$ - to $\beta$-phase is the slowest step, determining the rate of the overall hydrogen absorption process in C-Pd films.

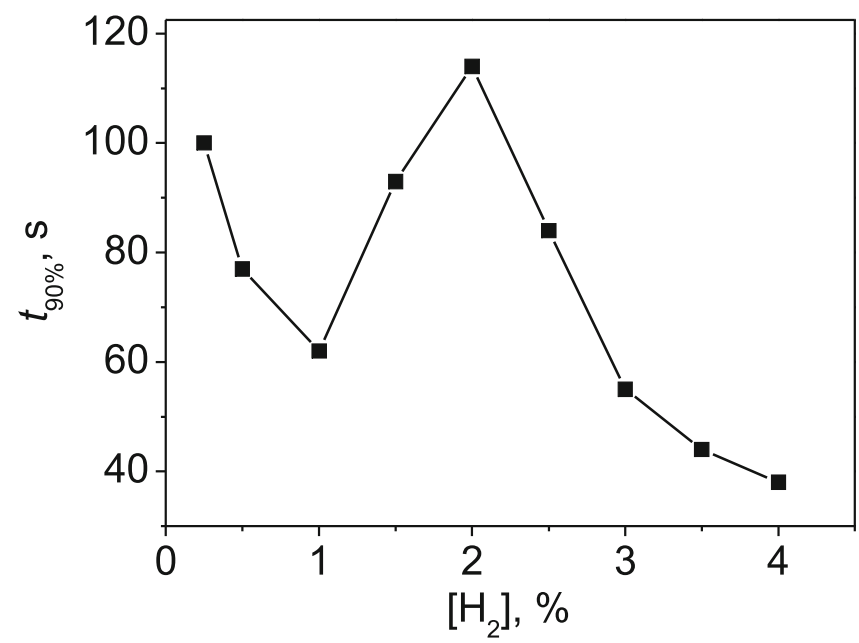

Figure 6. Response time of the C-Pd film in the function of hydrogen concentration

Summing up, the obtained results of changes in the sensitivity and response time of C-Pd layers are consistent with the results of the XRD studies. The presence of a small amount of hydrogen (less than 2 vol.\%) causes changes in the film resistance, but does not lead to a phase transition in the palladium nanograins. However, at higher hydrogen concentrations, a phase transition from $\alpha$ - to $\beta$-phase occurs, which is accompanied by an increase in the lattice constant of $3.5 \%$. The increase in the concentration of hydrogen (in the range of 2.5 vol.\%-4.0 vol.\%) does not cause a further increase in the film resistance. This means that the changes in the C-Pd film resistance are caused by the creation of $\alpha-\mathrm{PdH}_{\mathrm{x}}$ solid solution.

\section{CONCLUSIONS}

C-Pd films obtained in the PVD process, and then annealed in an argon flow are active in the reaction with hydrogen. In this paper, we found a correlation between changes in the C-Pd films crystal structure and electrical properties under the influence of hydrogen. At lower concentrations of hydrogen (up to 2 vol.\%), the formation of $\alpha-\mathrm{PdH}_{\mathrm{x}}$ solid solution results in a linear increase in the film resistance and a slight increase in the lattice constant with an increase of $\left[\mathrm{H}_{2}\right]$. Exceeding the hydrogen concentration of 2 vol.\% leads to a phase transition $\alpha \rightarrow \beta$, accompanied by a rapid increase in the lattice constant, while a further increase in the film resistance is not observed. The phase transition from the $\alpha$ - to $\beta$-phase affects the kinetics of the interaction of hydrogen with palladium, becoming the rate-limiting step of the overall hydrogen absorption process. It was shown that the changes of the C-Pd film electrical properties result from the creation of solid solution of hydrogen in the palladium. The hydrogen sensors based on such C-Pd films can find their application everywhere where hydrogen is used, due to danger of hydrogen leakage.

\section{ACKNOWLEDGMENT}

This project is co-financed by the European Regional Development Fund within the Innovative Economy Operational Programme 2007-2013 No. UDA-POIG.01.03.01-14-071/08-08 and Polish MNiSW Project No. W46/DESY/2012.

\section{LITERATURE CITED}

1. Zeng, X.Q., Latimer, M.L., Xiao, Z.L., Panuganti, S., Welp, U., Kwok, W.K. \& Xu, T. (2011). Hydrogen gas sensing with networks of ultrasmall palladium nanowires formed on filtration membranes. Nano Lett. 11, 262-268. DOI: 10.1021/nl103682s.

2. Topil'nikov, V.I. \& Sosna, M.K. (2012). Modeling paraffin hydrocarbon hydrocracking process. Chem. Technol. Fuels Oils 48(2), 135-142. DOI: 10.1007/s10553-012-0349-9.

3. Sheldon, R.A. \& Bekkum, H. van (2007). Catalytic Hydrogenation and Dehydrogenation. In Fine Chemicals through Heterogeneous Catalysis. Wiley-VCH Verlag GmbH, Weinheim, Germany. DOI: 10.1002/9783527612963.ch08.

4. Blaser, H.U. (2012). Industrial Asymmetric Hydrogenation. In M.L. Crawley and B.M. Trost (Eds.), Applications of Transition Metal Catalysis in Drug Discovery and Development: An Industrial Perspective. John Wiley \& Sons, Inc., Hoboken, NJ, USA. DOI: 10.1002/9781118309872.ch8.

5. Kumar, A., Zhang, P., Vincent, A., McCormack, R., Kalyanaraman, R., Cho, H.J. \& Seal, S. (2011). Hydrogen selective gas sensor in humid environment based on polymer coated nanostructured-doped tin oxide. Sens. Actuators B: Chem. 155, 884-892. DOI: 10.1016/j.snb.2011.01.065.

6. Noh, J.-S., Lee, J.M. \& Lee, W. (2011). Low-dimensional palladium nanostructures for fast and reliable hydrogen gas detection. Sensors 11, 825851. DOI: 10.3390/s110100825.

7. Joshi, R.K., Krishnan, S., Yoshimura, M. \& Kumar, A. (2009). Pd Nanoparticles and thin films for room temperature 
hydrogen sensor. Nanoscale Res. Lett. 4, 1191-1196. DOI: 10.1007/s11671-009-9379-6.

8. Yang, F., Kung, S.C., Cheng, M., Hemminger, J.C., Penner, R.M. (2010). Smaller is faster and more sensitive: The effect of wire size on the detection of hydrogen by single palladium nanowires. ACS Nano 4, 5233-5244. DOI: 10.1021/nn101475c.

9. Zilli, D., Bonelli, P.R. \& Cukierman, A.L. (2011). Room temperature hydrogen gas sensor nanocomposite based on Pd-decorated multi-walled carbon nanotubes thin films. Sens. Actuators B: Chem. 157, 169-176. DOI: 10.1016/j.snb.2011.03.045.

10. Hübert, T., Boon-Brett, L., Black, G. \& Banach, U. (2011). Hydrogen sensors - A review. Sens. Actuators B: Chem. 157, 329-352. DOI: 10.1016/j.snb.2011.04.070.

11. Lee, E., Lee, J.M., Koo, J.H., Lee, W., Lee, T. (2010). Hysteresis behavior of electrical resistance in Pd thin films during the process of absorption and desorption of hydrogen gas. Int. J. Hydrogen Energ. 35, 6984-6991. DOI: 10.1016/j. ijhydene.2010.04.051.

12. Xu, T., Zach, M.P., Xiao, Z.L., Rosenmann, D., Welp, U., Kwok, W.K., Crabtree, G.W. (2005). Self-assembled monolayer-enhanced hydrogen sensing with ultrathin palladium films, Appl. Phys. Lett. 86, 203104. DOI: 10.1063/1.1929075.

13. Yang, F., Taggart, D.K. \& Penner, R.M. (2009). Fast, sensitive hydrogen gas detection using single palladium nanowires that resist fracture. Nano Lett. 9, 2177-2182. DOI: 10.1021/n19008474.

14. Khanuja, M., Shrestha, S., Mehta, B.R., Kala, S. \& Kruis, F.E. (2011). Magnitude and time response of electronic and topographical changes during hydrogen sensing in size selected palladium nanoparticles. J. Appl. Phys. 110, 014318. DOI: $10.1063 / 1.3603053$.

15. Lee, J., Noh, J.S., Lee, S.H., Song, B., Jung, H., Kim, W., Lee, W. (2012). Cracked palladium films on an elastomeric substrate for use as hydrogen sensors. Int. J. Hydrogen Energ. 37, 7934-7939. DOI: 10.1016/j.ijhydene.2012.01.067.

16. Czerwosz, E., Diduszko, R., Dłużewski, P., Kęczkowska, J., Kozłowski, M., Rymarczyk, J., Suchańska, M. (2008). Properties of Pd nanocrystals prepared by PVD method. Vacuum 82, 372-376. DOI: 10.1016/j.vacuum.2007.08.003.

17. Kamińska, A., Krawczyk, S., Kozłowski, M., Czerwosz, E. \& Sobczak, K. (2013). Kinetics of interaction of hydrogen with nanostructured C-Pd films for hydrogen sensing. Sensor Lett. 11, 500-504. DOI: 10.1166/s1.2013.2915.

18. Ibañez, F.J. \& Zamborini, F.P. (2006). Ozone- and thermally activated films of palladium monolayer-protected clusters for chemiresistive hydrogen sensing. Langmuir 22, 9789-9796. DOI: $10.1021 / \mathrm{la} 0617309$.

19. Yang, F., Taggart, D.K. \& Penner, R.M. (2010). Joule heating a palladium nanowire sensor for accelerated response and recovery to hydrogen gas. Small 6(13), 1422-1429. DOI: 10.1002/smll.201000145. 